\section{ADALIMUMAB IN THE MAINTENANCE OF REMISSION FOR INFLAMMATORY BOWEL DISEASE: NEED FOR WEIGHT-BASED DOSING?}

G Chan, H Martin, R Farrell Gastroenterology Department, Connolly Hospital, Blanchardstown, Dublin, Ireland; RCSI, Dublin, Ireland

\subsection{6/gutjnl-2013-305143.42}

Introduction In inflammatory bowel disease (IBD), administration of adalimumab (ADA), unlike infliximab, is not weight based. Historically, patients with severe IBD, particularly CD are emaciated and typically have low body weight. Since the advent of biologic therapy, patients are started with aggressive therapy at an earlier stage of disease and most IBD patients are now of a normal or high body mass index (BMI). Epidemiological studies have demonstrated that the BMI of IBD patients have gradually increased over time. However, the impact of patient's weight on response to $\mathrm{ADA}$ is not known.

Aims/Background To evaluate the impact of patient's weight on the efficacy of ADA in maintaining remission in IBD.

Method All patients with IBD who received ADA were recruited retrospectively. Patients' weights prior to onset of ADA therapy were retrieved from file. Patients who did not achieve induction of remission with ADA were excluded. Remission was defined as a consistent Harvey Bradshaw score $<5$ for CD and Mayo score $</=2$ for UC. Treatment failure was defined as requiring escalation of therapy or surgical intervention.

Results $7.4 \%$ of patients (43/585) with IBD (31 CD, $12 \mathrm{UC}$ ) received ADA. Of these, 6/43 (14.0\%) patients were primary non responders while $3 / 43(7.0 \%)$ patients discontinued therapy due to side effects. Both these groups were excluded. In the 34 patients who responded to $\mathrm{ADA}$, there were equal numbers of male and female patients, with a mean age at diagnosis of $29.7 \pm 12.1$ years. The mean duration of ADA therapy was 25.4 months. Of those who initially responded to ADA, $11 / 34(32.4 \%)$ subsequently required escalation of therapy in the form of reduced dosing interval $(7 / 11)$, short term corticosteroid therapy (3/11) or surgical intervention (1/11). These patients who failed standard ADA therapy had a higher mean body weight of $79.6 \mathrm{~kg}$ compared to $70.7 \mathrm{~kg}$ in those who were successfully maintained in remission with fortnightly ADA, $\mathrm{p}=0.126$.

Conclusion Despite the higher mean body weight in IBD patients who fail ADA therapy compared to those who respond, the result did not achieve statistical significance. However, the study did demonstrate a trend towards treatment failure in patients with a higher mean body weight and would indicate that we should consider weight based adalimumab dosing. Other similar factors that may influence the need for weight based dosing include patients' BMI and abdominal girth. 